\title{
Villon entre mythe et poésie, textes édités par Jean Dufournet et Marcel Faure
}

\section{Alessandro Bertolino}

\section{(2) OpenEdition}

1 Journals

\section{Edizione digitale}

URL: http://journals.openedition.org/studifrancesi/3672

DOI: 10.4000/studifrancesi.3672

ISSN: 2421-5856

\section{Editore}

Rosenberg \& Sellier

\section{Edizione cartacea}

Data di pubblicazione: 1 décembre 2012

Paginazione: 547-548

ISSN: 0039-2944

\section{Notizia bibliografica digitale}

Alessandro Bertolino, «Villon entre mythe et poésie, textes édités par Jean Dufournet et Marcel Faure», Studi Francesi [Online], 168 (LVI | III) | 2012, online dal 30 novembre 2015, consultato il 05 mars 2021. URL: http://journals.openedition.org/studifrancesi/3672 ; DOI: https://doi.org/10.4000/studifrancesi. 3672

Questo documento è stato generato automaticamente il 5 mars 2021.

\section{(c) $($ ) $\odot$ EY}

Studi Francesi è distribuita con Licenza Creative Commons Attribuzione - Non commerciale - Non opere derivate 4.0 Internazionale. 


\title{
Villon entre mythe et poésie, textes édités par Jean Dufournet et Marcel Faure
}

\author{
Alessandro Bertolino
}

\section{NOTIZIA}

Villon entre mythe et poésie, Actes du colloque organisé les 15, 16 et 17 décembre 2006 à la Bibliothèque historique de la Ville de Paris par Michael FREEMAN, Jean DÉRENS et Jean DUFoURNET, textes édités par Jean DUfoURNET et Marcel FAURE, Paris, Champion, 2011, pp. 386.

1 Questo bel volume raccoglie gli atti di un convegno organizzato nel 2006 a Parigi da tre insigni studiosi villoniani, due dei quali recentemente scomparsi, con l'obiettivo di offrire nuove prospettive e spunti fecondi agli studi sull'opera del poeta. La raccolta si apre con un commosso ricordo di uno degli organizzatori, M. Freeman. Nel contributo iniziale Jean DUFOURNET (Quelques propos sur l'actualité villonienne, pp.11-15) segnala le ultime novità editoriali nel campo degli studi su Villon, così come i contributi 'non accademici' (libri di narrativa, film) che in tempi recentissimi il poeta ha ispirato.

Gli interventi si articolano intorno ad alcune grandi aree tematiche; una delle più importanti è il rapporto tra lirismo personale e satira. Nei contributi di Michael EDWARDS (Villon et la cloche de Sorbonne, pp. 19-29) e di Marcel faURE (Promenade dans l'entre-deux de François Villon, pp.181-185), la finzione testamentaria è vista rispettivamente come strumento per lasciar traccia di sé esercitando nel contempo una certa forma di potere attraverso l'atto derisorio dell'eredità da assegnare, e come modo per giustapporre modalità di scrittura apparentemente contraddittorie al fine di rendere la scoperta dell'opera più ardua, per affrontare la quale il lettore è costretto a crearsi una strategia di lettura. Se per Elisabeth GAUCHER (Emblèmes et devises dans le Lais et le Testament de Villon, pp. 33-43) la rappresentazione dei lasciti, in cui la definizione 
delle eredità da assegnare ad ogni legatario ricorda la forma dell'emblema, permette di ricostruire un interessante quadro della vita cittadina dell'epoca, per Luca PIERDOMINICI (La médisance entre affabulation et poésie, pp.166-178) questa modalità di scrittura permette di attaccare i nemici con ironia, senza maldicenza, ma creando una 'rete' dalla quale i destinatari non possono liberarsi se non accettando di buon grado queste eredità. Bertrand DEGOTT (Villon et la ballade, pp. 91-103) riflette poi sulla forma della ballata, identificandovi una "structure rayonnante» che si diparte dal ritornello e si caratterizza per gli effetti di accumulazione creati dal persistere degli schemi ritmici.

Un secondo gruppo di interventi si concentra su aspetti particolari dell'opera villoniana o su singoli componimenti; l'elemento comune è qui l'analisi di temi e immagini dal valore contraddittorio e volutamente ambiguo: la rappresentazione dell'immaginario carcerario per deridere l'immagine della «prigione d'Amore» e la corrente bucolica (Fleur VIGNERON, 'Enserrez soubz trappe voliere': la prison dans l'œuvre de François Villon, pp. 63-78), l'evocazione della cavalleria tra derisione e rimpianto (Claude BACHET, Villon et la chevalerie: entre démythificaton et nostalgie, pp. 47-59), l'uso dei temi della «mort d'amour» e del martirio d'amore sia con intento parodico che come omaggio ammirato all'opera di Alain Chartier (Jean DEVAUX, Villon et 'La Belle Dame sans mercy', pp. 201-215). La compresenza di ironia e melanconia, nonché la grande ricchezza di nuances tematiche che spesso non trovano spazio nella interpretazione vulgata emergono con chiarezza dalle analisi di due ballate celebri (Philippe MÉNARD, Réflexions sur la 'Ballade des Dames du temps jadis' de Villon, pp. 107-124; Jean DUfOURNET, Au cœur du Testament de Villon: la Ballade pour Robert d'Estouteville, pp.151-162); in particolare, nella ballata dedicata a Robert d'Estouteville, le immagini del lauro e dell'ulivo sembrerebbero evocare, oltre all'influenza pacificatrice della moglie nel matrimonio, anche l'immagine di una poesia più colta, elaborata e dotta. I contributi di Michael FREEMAN (Mais pourquoi 'le tiers Calixte'?, pp. 81-86) e di Christine MARTINEAU-GÉNIEYs (Villon l'imposteur, pp.127-148) tentano di giustificare la presenza di due personaggi storici citati nel Testament, rispettivamente papa Callisto III nella Ballade des seigneurs e la bella Archipiades nella Ballade des dames: Callisto III in exergo alla ballata permetterebbe di evocare Giovanna d'Arco, dal momento che uno dei primi gesti del neo-eletto papa fu proprio quello di autorizzare la revisione del processo della Pulzella, mentre l'inserimento di Alcibiade all'interno di una rassegna di dame del passato sarebbe dovuto alla volontà di nascondere, attraverso un complesso gioco di anagrammi, il nome di un amante, Ytier Marchant. Jean-Pierre Lemaire ( $L a$ voix et l'épitaphe, pp. 189-197) si concentra sulla celebre Ballade des pendus, sottolineando la funzione di una scrittura che abbandona ogni carattere allusivo per comunicare un messaggio di fratellanza universale.

4 Una terza parte riunisce una serie di contributi dedicati alla costruzione del «mito Villon» e, più in generale, alla ricezione e alla fortuna della sua opera presso i suoi contemporanei, ma anche presso le generazioni successive. Un primo esempio della costruzione di una mitologia del personaggio Villon fondata sugli aspetti più popolari e grotteschi della sua opera è il Recueil des repues franches de Maistre François Villon (Nelly LABÈRE, Les compagnons des 'Repues Franches' (1480-1500) à la table de Maistre François Villon, pp.251-260), in cui si gioca abilmente con il discorso religioso realizzando un'inversione di valori che assicurerà la prosperità letteraria del personaggio Villon, rappresentato qui come un maestro in grado di sfamare i suoi discepoli insegnando loro tutti i trucchi per scroccare un pranzo; la sua rappresentazione stereotipata entra 
velocemente nella cultura dell'epoca, come dimostrano anche le incisioni dell'edizione Levet (1489) studiate da Jean-Marie PRIVAT (Villon le bestorné, pp. 293-311), in cui la raffigurazione di Villon mostra aspetti inquietanti (il piede sinistro storpio, la mano sinistra alzata in segno di giuramento, lo sguardo torvo) e ricorda da vicino la figura del saltimbanco nel mazzo dei tarocchi. Il poeta ricompare come personaggio, come è noto, anche alla fine del Quart Livre di Rabelais; Pierre Johan LAFFITTE (Maistre François (Villon), figure exemplaire dans le 'Quart livre' de Maistre François (Rabelais), pp. 263-276) mostra che nell'episodio in cui il poeta è rappresentato alla corte di Edoardo $\mathrm{V}$ d'Inghilterra viene messo in scena un «maestro» nell'arte dell'interpretazione, che svela la verità al potente e che, per non incorrere nelle ire del re, non esita a sminuire la portata della propria parola rivelatrice, descrivendosi come un semplice badault, uno sciocco; Estelle DOUDET (Villon et les Réthoriqueurs: mythologie comparée de l'automne du Moyen Age, pp. 219-233) ricostruisce i legami che hanno unito le fortune di Villon e dei Réthoriqueurs, a partire dai Robertet che furono i primi a sottrarre la figura di Villon al mito del «bon follastre», fino al XIX secolo, epoca in cui il personaggio di Gringoire nelle opere di Hugo e Théodore de Bainville suscita un rinnovato interesse sia per il poeta che per la Grande Réthorique. Il più importante tra coloro che contribuirono al mito di Villon è però senza dubbio Clément Marot; la genesi della sua edizione delle Cuvres de Françoys Villon (1533) è l'oggetto della comunicazione di Claude thIRY (Marot, éditeur de Villon, pp. 281-290), che si interroga soprattutto sul testo di cui l'editore si è servito come base, ipotizzando l'esistenza di un manoscritto oggi perduto. La produzione di Henri Baude, poeta meno conosciuto, ma che può ritenersi un emulo di Villon, è al centro del contributo di Marie BouHAïK-GIRONÈs (François Villon et Henri Baude, pp. 237-247), secondo la quale i punti di contatto tra i due poeti andrebbero ricercati nella cultura della Basoche, che diede vita a una letteratura popolare incentrata su temi triviali, ma nobilitata da sofisticati giochi linguistici.

5 Infine, tre lavori fanno riferimento ad alcune riscritture di Villon. Jane TAYLOR (Les 'Villoneries' d'Algernon Charles Swinburne: Villon traduit par un pré-raphaélite, pp. 321-330) fa notare come le traduzioni di Swinburne abbiano mostrato alla società vittoriana del tempo un medioevo diverso, mentre nella Ballad of François Villon il poeta inglese proclama una fratellanza tra se stesso e Villon. Robert PECKAM (Mythamorphose des dames du temps jadis dans la littérature américaine, pp. 333-343) passa in rassegna una serie di autori americani che si sono in varie modalità appropriati del modello stilistico della Ballade des dames, adattandolo alle proprie esigenze espressive e ai temi più vari. L'ultimo tra questi contributi ricostruisce l'esperienza particolare di un lettore moderno di Villon, l'intellettuale e regista Guy Debord (Boris DonNÉ, Debord lecteur de Villon, pp. 347-366): attraverso un'accurata analisi dei riferimenti a Villon, l'A. ci restituisce il ritratto di un Debord che apprezza il poeta medievale per la sua vita movimentata, ma soprattutto per la sua capacità di raccontare la fugacità della bellezza e la precarietà di ogni esistenza. La lettura di Villon permette inoltre a Debord di trovare modelli per la sua pratica di scrittura.

Una particolare segnalazione merita la scelta di inframmezzare i vari contributi con delle ballate, scritte alla maniera di Villon tra il 1870 e il 2000, testimoniando così, se ce ne fosse bisogno, della grande modernità di questo poeta. 